\title{
RÉSULTATS D'UN MARQUAGE DE TRUITES SUR LE LAC D'ANNECY
}

\author{
par Georges GRIVAZ \\ Ingénieur des Eaux et Forêts.
}

\section{L'opération DE MARQUAGE.}

En 1957, l'Administration des Eaux et Forêts nous a ouvert un crédit (provenant, par fonds de concours, de redevances piscicoles de l'E. D. F.) pour alevinage d'automne en Truitelles dans le lac d'Annecy.

Dans sa lettre annonçant ce crédit, l'Administration ajoutait que, si les Associations de pêcheurs du lac d'Annecy voulaient bien récompenser ceux qui leur signaleraient les Truites marquées qu'ils auraient prises, il serait intéressant de marquer ces Truitelles.

Les Associations en question ayant donné leur accord pour ces récompenses (qui furent fixées à 300 francs par Truite reprise) l'opération fut décidée.

Nous passons sur les détails de son organisation, nous contentant de signaler qu'elle fut accompagnée d'une certaine publicité sous forme d'un "Avis aux pêcheurs " qui fut épinglé à chaque permis de pêche aux filets et à chaque permis de pêche "à la sonde et à la traîne " sur le lac d'Annecy, et qui fut reproduit (gracieusement) par la Presse locale.

La pince à marquer et les marques métalliques numérotées (à fixer à la mâchoire inférieure des Truitelles) nous furent envoyées par la Direction générale des Eaux et Forêts.

L'opération fut faite le 19 Novembre 1957, sous notre contrôle, à la pisciculture domaniale de Menthon Saint-Bernard par le Chef de District à Menthon (poste de Pêche) avec la collaboration du GardeChef et de trois gardes de la Fédération départementale de Pêche. Le Garde-Chef Boucheron à Thonon (du Conseil Supérieur de la Pêche) vint assister au début de l'opération pour achever d'initier le Chef de district de Menthon à la technique du marquage : dose d'anesthésiant à utiliser pour endormir les poissons $(3$ à 4 grammes d'uréthane pour un litre d'eau), durée moyenne d'immersion dans la solution d'uréthane (quelques minutes), façon d'apposer la marque métallique d la machoire inférieure des poissons, sans gêner la langue. 
Il fut vite constaté qu'il était pratiquement impossible, avec ce procédé de marquage, d'opérer sur des Truitelles de moins de 12 centimètres de longueur. En-dessous de cette taille, on risquait trop de déchirer gravement la mâchoire du poisson, de lui prendre le frein de la langue dans la marque, etc. (1).

Il n'était d'ailleurs pas question de marquer toutes les 5.000 Truitelles à déverser dans le lac d'Annecy à l'automne 1957. Les " estivaux " de 6-12 centimètres furent donc déversés sans marque; seuls 940 poissons de 12 à 22 centimètres de longueur (433 Truitelles Fario, 505 Truitelles Arc-en-ciel et 2 Saumons de fontaine) furent marqués avant d'être déversés en différents points du lac d'Annecy (à Menthon, à Veyrier, à Talloires).

Ces 940 poissons provenaient d'une pisciculture privée locale (pisciculture Fournier à Vieugy). Les "Fario " avaient une taille moyenne de 17,19 centimètres et un poids moyen de 50,7 grammes; elles étaient âgées de 2 étés ou de 3 étés. Les " Arc-en-ciel " avaient une taille moyenne de 18,8 centimètres et un poids moyen de 66,9 grammes; elles étaient âgées de 2 étés.

\section{Capture des Truitelles marquées.}

Bien entendu, chaque Truitelle marquée avait fait l'objet d'une inscription sur un registre avec le no de la marque, sa taille, son poids et les observations éventuelles sur son état de santé.

Nous devons signaler ici l'intérêt réel qu'ont montré les pêcheurs pour cette expérience. En apportant leur prise aux adresses indiquées sur notre " avis aux pêcheurs", ils demandaient où, quand, et à quelle taille ce poisson avait été mis à l'eau. Chaque fois une lettre de remerciement fut adressée par nos soins au pêcheur ayant capturé une de ces Truites marquées, lettre donnant tous ces renseignements et dont une copie était envoyée à leur Association en vue du versement de la prime de 300 francs par capture (les pêcheurs professionnels ont d'ailleurs fait remise gracieuse du montant de ces primes à leur Association).

Si bien qu'avant que nous ayons nous-même dépouillé et tiré parti des résultats, tous les pêcheurs savaient que les Truites prenaient au moins 40 grammes par mois dans le lac d'Annecy!

Les premières Truites marquées reprises entre l'ouverture de la pêche (le 1er Février 1958) et le 21 Mars 1958, furent au nombre de 11 , dont une seule dans le lac, les 10 autres dans les petits affluents du lac; il s'agissait de poissons mis à l'eau avec une taille de 20 centimètres environ et qui, vraisemblablement, étaient remontés dans les ruisseaux

(1) Nous avons d'ailleurs observé par la suite que sur 26 Truitelles de $13 \mathrm{~cm} .5$ et au-dessous marquées et déversées dans le lac, aucune ne fut retrouvée (on nous en a signalé une cependant reprise en Février ou Mars 1958 et portant la trace de la marque métallique qui s'était détachée) alors que, comme nous le verrons, beaucoup de Truitelles marquees de $13 \mathrm{~cm}$. 6 et au-dessus furent reprises. 
pour leur première reproduction ('un d'entre eux, un mâle Arc-en-ciel, fut retrouvé perdant sa laitance le 9 Février 1959, dans le ruisseau de Duingt à 2 kilomètres de Talloires, lieu de son déversement dans le lac). Sur ces 10 Truites, 6 étaient des Arc-en-ciel, 4 des Fario, proportion comparable à celle des déversements (505 Arc-en-ciel pour 433 Fario).

Après le 21 Mars 1958 les prises eurent lieu surtout dans le lac d'Annecy, si bien qu'entre cette date et la fermeture de la pêche aux Truites en 1958 (mi-Octobre) sur 92 captures de Truites marquées, seules 9 furent faites dans les affluents du lac (et encore dans la zone calme de ces rivières, à proximité de leur embouchure), toutes les autres eurent lieu dans le lac, et surtout pendant les mois de Juin, Juillet et Août (période de pêche active pour les pêcheurs aux filets). Remarquons tout de suite que sur les 9 Truites pêchées en rivière pendant cette période, 4 sont des Arc-en-ciel et 5 des Fario, ce qui montre qu'un certain nombre de Truitelles déversées dans le lac remontent et demeurent en rivière (au moins dans la zone relativement calme de celle-ci), et que parmi ces Truitelles émigrées, les Fario sont au moins aussi nombreuses que les Arc-en-Ciel.

Enfin, après la période de fermeture de la pêche aux Truites (du 15 Octobre 1958 au 31 janvier 1959) 5 Truites marquées ont encore été prises et signalées, entre Février et Avril 1959 dont 2 en rivière et 3 dans le lac (1).

\section{Résultats.}

Chaque capture qui a été accompagnée de renseignements certains et précis (c'est le cas de la quasi-totalité des captures) a été enregistrée et on en a tiré un point sur des graphiques où sont portés :

— en abcisse : le temps écoulé depuis le déversement du 19 Novembre 1957 ;

- en ordonnée : le poids des poissons capturés.

Ces graphiques sur lesquels nous avons donc pu ensuite tracer la courbe de croissance en poids sont au nombre de 4 :

1 pour les Truites "Fario " capturées dans le lac;

1 pour les Truites "Arc-en-ciel " capturées dans le lac;

1 pour les Truites "Fario " capturées en rivière;

1 pour les Truites "Arc-en-ciel " capturées en rivière.

Le premier graphique (p. 25), est celui qui, de beaucoup, comporte le plus de points, et c'est le seul qui intéresse réellement le

(1) Notons aussi à titre de curiosité, qu'en ce qui concerne les deux Saumons de fontaine glissés par erreur dans un lot de Truites et que nous avions cependant marqués et mis au lac le 19 Novembre 1957, l'un d'eux a été repris le 5 Juin 1958 dans la Bornette à 1 kilomètre de l'embouchure de cette petite rivière affluente du lac. Rappelons à ce sujet que des essais d'acclimatation de Saumons de fontaine furent entrepris il y a une quinzaine d'années dans le lac d'Annecy et n'ont abouti à rien. 
lac d'Annecy. Les autres graphiques ne font que donner des indications comparatives qui ne manquent cependant pas d'intérêt.

Nous avons pu aussi tracer de la même façon, la courbe de croissance en longueur des Truites dans le lac.

QUelle SONT LES OBSERVATIONS QUe L'ON PEUT FaIRe SUR CES RÉSULTATS ?

$1^{0}$ Observations sur les pourcentages de reprise :

103 Truites marquées ont été capturées dans les 16 mois qui ont suivi leur déversement; ceci représente un pourcentage global de reprises

$$
\text { de } \frac{103}{938}=10,98 \% \text {. }
$$

La plupart de ces Truites ont été capturées dans le lac (84, soit $8,96 \%$ des Truites déversées) et les autres $(19$, soit $2,02 \%$ ) dans les petites rivières affluentes du lac.

Certes la publicité sur le marquage à moins touché les pêcheurs en rivière que les pêcheurs du lac (les premiers ayant été avisés par la presse seule, alors que les seconds l'ont été à la fois par la presse et par un avis joint à leur permis); de sorte que le pourcentage de capture en rivière est peut-être moins significatif que celui des captures dans le lac.

Mais une première observation peut cependant être faite :

- C'est que, contrairement à ce que certains croyaient, la plupart des Truitelles déversées dans le lac demeurent dans celui-ci : beaucoup remontent en rivière à l'époque de la reproduction pour revenir au lac ensuite, peu remontent en rivière pour y demeurer.

En effet, nous avons vu que 10 Truitelles marquées ont été reprises en rivière en moins de 2 mois, du 1 er Février au 21 Mars 1958, au moment même où une seule reprise avait lieu dans le lac ; mais seulement 9 Truitelles marquées ont été reprises en rivière pendant près de 7 mois, entre le 21 Mars 1958 et la mi-Octobre 1958, au moment même où 92 reprises avaient lieu dans le lac.

Une deuxième observation peut aussi être faite :

- C'est que les Truites "Fario "s'acclimatent bien et restent dans le lac d'Annecy, alors que l'on peut douter de la réussite des Truites "Arcen-ciel ".

En effet, sur 85 Truites marquées capturées dans le lac d'Annecy, 73 sont des Fario et 12 des Arc-en-ciel, ce qui représente les pourcentages suivants :

Fario..............

Déversées
433
505

Reprises
85
12

$$
\begin{gathered}
\% \\
19,63 \\
2,37
\end{gathered}
$$

Arc-en-ciel .........

505

Comme les pourcentages de capture en rivière sont à peu près les mêmes pour les Fario et les Arc-en-ciel :

$\begin{array}{lrrr}\text { Fario............ } & 433 & 9 & 2,08 \\ \text { Arc-en-ciel } \ldots \ldots \ldots & 505 & 12 & 2,37\end{array}$


$-25-$

Courbe de croissance en poids des Truites "Fario" dans le lac d'Annecy.

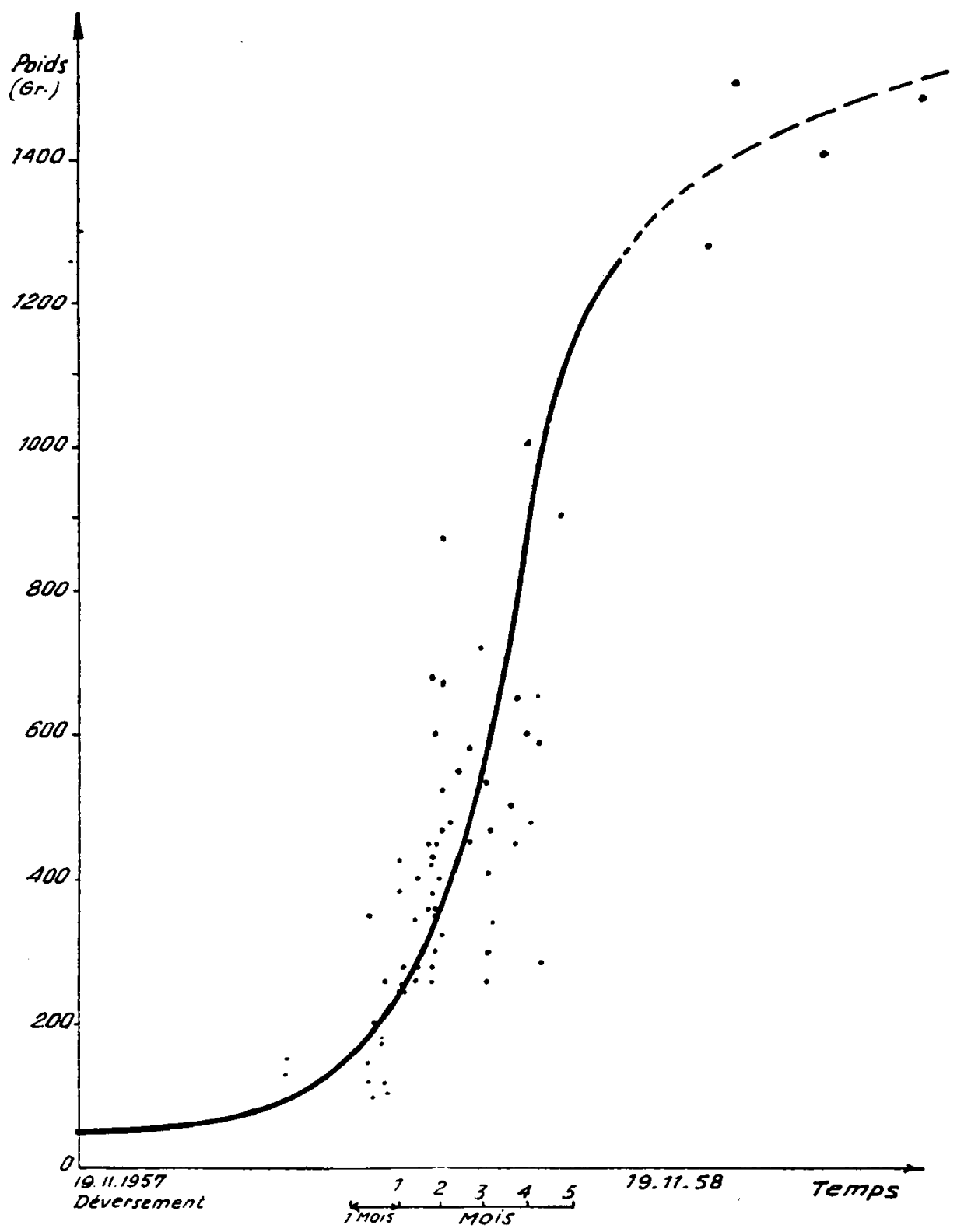


il faut en conclure que ces Truites "Arc-en-ciel " ne se retrouvent presque plus dans le lac d'Annecy sans pour autant remonter plus en rivière que les "Fario ". Leur mortalité est-elle donc très forte dans le lac? (Nous verrons cependant que celles qui survivent y croissent très vite, encore un peu plus vite que les "Fario "). Perdent-elles leur marque de mâchoire plus facilement que les Fario? (Ces Arc-en-ciel avaient cependant 2 étés et leur mâchoire était déjà bien ossifiée). Migrent-elles vers l'aval du lac jusque dans le Fier où elles avaient peu de chances d'être reprises par des pêcheurs avertis de l'opération? (2 Arc-en-ciel marquées ont été effectivement reprises à l'aval du lac dans le Fier jusqu'à Rumilly). Quoiqu'il en soit, le fait est qu'elles ne semblent guère réussir dans ce lac; ce fait était déjà plus ou moins connu localement, car quelques essais d'alevinages avec des Truites arc-en-ciel faits dans le passé au lac d'Annecy n'avaient pas donné de résultats visibles.

Enfin une troisième observation :

- Le pourcentage élevé de captures dans le lac $(19,63 \%$ pour les Truitelles Fario, en 16 mois) montre combien la pêche y est intensive.

$2^{\circ}$ Observations sur la croissance des Truites :

De temps en temps, il se prend (au filet) dans le lac d'Annecy une Truite énorme (pesant jusqu'à $15 \mathrm{~kg}$ !). Les avis des pêcheurs étaient très variés en ce qui concerne l'âge de ces poissons, certains parlant de plus de 30 ans!

L'an dernier (au mois d'Octobre 1958) nous avions pu étudier une de ces truites. Elle pesait $8 \mathrm{~kg} 300$ et avait une longueur de 87 centimètres.

Les écailles de ce poisson étaient claires et, observées avec un appareil de projection, montraient des zones d'accroissement annuel très larges et nettes; son âge ainsi déterminable avec assez de précision (à 1 an près étant donné certaine imprécision sur la première zone annuelle d'accroissement) était de 6 ou 7 ans.

Cette constatation qui semblait étonnante et pouvait faire penser que l'on était en face d'une Truite absolument exceptionnelle (ou bien que l'on s'était trompé en analysant les zones d'accroissements annuels) ne fait cependant que confirmer les résultats obtenus par le marquage :

La courbe de croissance en poids des Truites "Fario " déversées puis capturées dans le lac d'Annecy (courbe assez facilement tracée entre 73 points) montre en effet que la croissance de ces Truites dans le lac a été en moyenne de 500 grammes dans les 9 mois qui ont suivi le déversement et de plus de 1.000 grammes en un an, du 19 Novembre 1957 au 19 Novembre 1958.

Les courbes de croissance en poids des Truites arc-en-ciel dans le lac d'Annecy ainsi que des Truites remontées dans les petits affluents du lac, sont bien moins significatives, car elles sont tracées avec un nombre de points plus faible, Elles donnent cependant des ordres de grandeur intéressants : c'est ainsi que les Truites arc-en-ciel qui restent dans le lac (nous avons vu qu'elles sont rares) semblent s'accroître encore un peu plus vite que les Fario! 
Quant aux Truites remontées et demeurées en rivière (dans le cours inférieur assez calme de ces cours d'eau, rappelons-le) on peut admettre (en " extrapolant" quelque peu les courbes) une croissance annuelle de 220 à 330 grammes (les Fario semblant s'accroître aussi vite que les Arc-en-ciel).

Quelques mots maintenant sur la croissance en longueur :

Le graphique de croissance en longueur des Truites Fario du lac montre qu'elles ont grandi de 20 à 23 centimètres en moyenne entre le 19 Novembre 1957 et le 15 Octobre 1958. Ainsi, au 15 Octobre 1958, au moment de la fermeture de la pêche, ces Truites ayant en général 3 étés (4 étés pour certaines) c'est-à-dire l'âge où elles commencent à se reproduire efficacement, avaient une taille d'environ 40 centimètres.

La taille minimum réglementaire des Truites pêchées dans le lac devrait donc théoriquement être, pour le moment, de 40 centimètres ! Or elle n'est que de 23 centimètres.

Heureusement beaucoup de Truites qui se prennent dans le lac ont bien 40 centimètres (grâce notamment aux dimensions imposées pour les mailles des filets).

Il n'est dependant pas question de proposer l'augmentation à 40 centimètres de cette taille réglementaire, car cette mesure qui ne pourrait d'ailleurs être prise qu'en obtenant une dérogation au décret du 16 Septembre 1958 modifie en Janvier 1959, serait difficilement applicable tout en n'ayant qu'un intérêt restreint. En effet, si elles atteignaient en grand nombre l'âge de la reproduction, les Truites du lac d'Annecy ne disposeraient pas de frayères en suffisance.

Il n'en demeure pas moins que cette observation sur l'insuffisance théorique de la taille réglementaire jointe à l'observation sur l'intensité de la pêche nous conduisent à ne compter guère que sur un alevinage intensif pour développer, puis maintenir la population de Truites du lac d'Annecy.

\section{Conclusion.}

La rapidité de croissance et l'excellente adaptation des Truites fario dans le lac d'Annecy montrent qu'il y a une place importante, une véritable "niche "à remplir.

En observant les mœurs des "poissons nobles " de ce lac (Truite, Corégones, Omble chevalier) on remarque :

a) Que les Truites ne se tiennent pas dans les mêmes zones que les Ombles chevaliers qui nagent en général dans les couches profondes du lac. Ces deux espèces ne semblent donc pas devoir entrer en concurrence entre elles, du moins si l'on se maintient dans les limites raisonnables de densité de population, limites dont nous sommes pour l'instant fort loin (1).

(1) De toute façon, l'Omble chevalier semble condamné à diminuer d'importance dans le lac d'Annecy, par suite d'une pollution lente, mais certaine, des eaux Qu'il soit remplacé progressivement par la Truite n'est pas un mal. 
b) Que les Truites se tiennent $\dot{a}$ peu près dans les mêmes zones que les Corégones (couches de profondeur moyenne ou même, en hiver, couches voisines de la surface) mais elles ne peuvent entrer en concurrence alimentaire avec les Corégones étant donné qu'elles n'ont pas, dans l'ensemble, la même nourriture. Cependant nous pouvons craindre une certaine prédation des tout-jeunes Corégones par les Truites au mois de Février; en effet, Février est le mois où, malgré les nombreuses larves d'Éphéméroptères du lac, qui arrivent à éclore même en plein hiver par les belles journées, la nourriture se fait relativement rare pour les Truites qui commencent à vouloir s'alimenter copieusement après leur période de reproduction; en Février elles se déplacent donc beaucoup dans la couche voisine de la surface où se tient également, à cette époque, du zooplancton; or c'est le moment où dans cette même zone, commencent à éclore et à se déplacer les minuscules alevins de Corégones.

Mais la prédation massive des tout-jeunes Corégones est déjà un fait réalisé par les petites Perches, si nombreuses dans cette zone, et nous ne pensons pas que cette prédation augmentera d'une façon sensible par suite de l'augmentation du nombre de Truites, elles-mêmes d'ailleurs prédatrices de Perchettes.

Déjà M. le Conservateur des Eaux et Forêts Hubault, professeur à l'École Nationale des Eaux et Forêts, écrivait il y a une quinzaine d'années (Annales de l'École Nationale des Eaux et Forêts, Tome X, fascicule 2, 1947, page 245) que le lac d'Annecy est essentiellement un lac $\grave{a}$ Truites. Il fondait cette opinion sur les constatations suivantes : Altitude forte $(447 \mathrm{~m}$.), eaux froides et suffisamment oxygénées aux profondeurs moyennes, Cyprinidés en nombre suffisant pour fournir la nourriture nćcessaire... Et, $M$. Hubault regrettait que l'on n'ait pas cherché à favoriser l'extension des Truites communes dans le lac.

En fait, depuis plusieurs années, l'avis de M. Hubault a commencé à être suivi : 300.000 à 400.000 alevins de Truites communes y sont déversés annuellement. Mais ils sont déversés très jeunes (à la résorption de la vésicule en général) et la quantité extrêmement importante des Perches (prédatrices de petites Truitelles) fait douter de l'excellent rendement de ces déversements qui ont cependant commencé à faire croître le tonnage des Truites pêchées.

Le résultat des marquages de 1957 nous a montré, par contre, le rendement étonnant des déversements de Truites de plus de $13 \mathrm{~cm}$. 5 de longueur. Quant à celles de 6 à $13 \mathrm{~cm}$. 5, nous n'avons pas de preuves chiffrées, mais les dires des pêcheurs d'une part (la pêche aux Truites a été meilleure en 1959 que lors des années précédentes et les pêcheurs prétendent, en se basant sur la teinte sombre de ces Truites, qu'il s'agit des estivaux déversés en 1957), d'autre part la vigueur avec laquelle nous avons vu nager (et échapper aux Perches) la plupart des estivaux que nous avons fait mettre au lac depuis 1957, font bien augurer du rendement de leur déversement.

C'est pourquoi, après 5.000 estivaux à l'automne 1957, l'Administration nous permit d'en déverser, toujours à l'aide des mêmes fonds, 
7.000 à l'automne 1958, puis 10.000 à l'automne 1959 (auxquelles s'ajoutent 5.000 estivaux déversés aux frais des deux Associations de pêche et de pisciculture du lac d'Annecy encouragées par les résultats du marquage).

La plupart de ces estivaux proviennent de l'établissement fédéral de Vermenoux; nous pensons poursuivre ces alevinages dans les mêmes conditions jusqu'à ce que la Pisciculture de la ville d'Annecy (1) soit en état de fournir annuellement, moitié pour le lac, moitié pour les rivières du Secteur d'Annecy, au moins 250.000 Truitelles communes de 10 mois.

(1) La Pisciculture de la ville d'Annecy, que feront fonctionner les deux Associations locales de pècheurs amateurs, a fait l'objet en 1959 d'un prêt de l'État à la Ville d'Annecy. Elle va être construite à côté de l'usine des eaux potables de la Ville, qui pompe l'eau à 38 mètres de profondeur dans le lac, puis la filtre ; et c'est l'abondant plancton ainsi récupéré sur les filtres qui servira au grossissement jusqu'à 10 mois de quelques centaines de milliers d'alevins. 